\title{
Tratamiento cognitivo conductual de la migraña en el adulto
}

\author{
María Elena Navarro Calvillo \\ Facultad de Psicología, Universidad Autónoma de San Luis Potosí, México. \\ Dirección postal: Paseo de los fresnos \#106 Colinas del parque. C.p. 78060. \\ Ce: maelenanavarro@hotmail.com
}

Resumen. El dolor crónico de la migraña es uno de los síndromes más comunes, caracterizado por dolores de cabeza recurrentes, el cual afecta al 25\% de la población. El dolor es un fenómeno perceptivo que necesita un enfoque multidimencional. En la migraña, además de tener una explicación neurológica, se han encontrado correlaciones con la calidad de vida, el trabajo, el estrés o componentes de tipo psicosocial. Se han empleado en las últimas décadas tratamientos cognitivo-conductuales combinados con técnicas de relajación y retroalimentación, los cuáles han aportado resultados que aunque no del todo contundentes, son una alternativa importante para los pacientes con migraña. En este artículo se analizan las características del dolor crónico y los factores psicológicos asociados, para después hacer una revisión sobre el dolor crónico de la migraña y la efectividad del tratamiento cognitivo conductual.

Palabras clave: dolor crónico, migraña, variables cognitivas, tratamiento cognitivo conductual.

\begin{abstract}
The chronic pain migraine is one of most common of the syndromes characterized by recurrent headaches, which affects $25 \%$ of the population. The pain is a perceptive phenomenon that needs a multidimensional approach. In migraine a neurological explanation have a role, but also factors like the quality of life, work, stress or component of psycho-social type have been correlated. Combined cognitive behavior treatments with relaxation techniques and feedback have been used in the last decades which have contributed forceful results that although not at all, are an important alternative for the patients with migraine. In this article, the psychological characteristics of the chronic pain and factors associate are analyzed, later to make a revision on the chronic pain migraine and the effectiveness of the behavior cognitive treatment.
\end{abstract}

Key Words: Chronic pain, migraine, cognitive variables, behavior cognitive treatment. 


\section{Introducción}

El dolor es una experiencia de tipo perceptivo, que todas las personas de todos los niveles socioeconómicos, culturales, étnicos o de género, van a sufrirlo en un momento de su vida. Es una experiencia personal, privada, influenciada por múltiples factores (experiencias pasadas, padecimiento, valor asignado culturalmente al dolor, aspectos psicológicos, psicofisiológicos, cognitivos, afectivos, etc.). En conclusión, el dolor es una experiencia influenciada por factores biológicos y psicosociales. Cuando el dolor es crónico, afecta de manera negativa a las actividades laborales, de ocio o pasatiempos, en la productividad y en general en las relaciones. El impacto social de la migraña puede ser asociado no sólo en términos directos al gasto de medicamentos, sino también en términos indirectos al costo de la falta de productividad. La migraña es responsable de más de 112 millones de días por año de personas postradas en una cama por el dolor. Las pérdidas por reducción de productividad en el empleo son de $\$ 13$ billones de dólares por año (Stephen, Silberstein y Lipton, 2001).

Los pacientes con dolor crónico no experimentan únicamente malestares físicos, sino también cambios significativos en su vida diaria, como disminución de su actividad y productividad, trastornos del sueño, problemas en sus relaciones sexuales y familiares, depresión y sentimientos de minusvalía (Turk y Fernández, 1997). Los pacientes con migraña pueden tener asociadas otras alteraciones como trastornos de sueño, estrés, ansiedad, depresión entre otros y es importante analizar la comorbilidad para ofrecer un diagnóstico y tratamientos más oportunos.

La migraña tiene explicaciones neurológicas, hormonales y está asociada con apoplejía, epilepsia y muchos otros trastornos emocionales. Los factores psicosociales que determinan a la migraña han sido ampliamente asociados con el dolor de cabeza. Estos incluyen estilos de vida, afectividad negativa, ansiedad o depresión. Principalmente, la afectividad negativa y por consecuencia el estrés, son factores muy asociados al dolor crónico (Tallegen, Lyken y Bouchard, 1984). Algunos eventos de la vida como divorcios, muerte de un familiar, desempleo, cambio de lugar de vivienda, etc.; producen mayor estrés y consecuentemente mayor predisposición a sufrir dolores de cabeza. Estas personas por lo general, tienen un ajuste negativo a estas situaciones (Kohler y Haimerl, 1990). Estos eventos son más frecuentes a partir de los 30 años y la prevalencia de migraña en mujeres es más alta que en los hombres, en edades entre 25 y 55 años, en los que se asocian un mayor número de factores biológicos, psicológicos y sociales.

El apoyo en los factores psicosociales es esencial para el manejo del dolor crónico de la migraña. Existen varios tratamientos y técnicas 
psicológicas como son: apoyo psicológico de grupo, condicionamiento operante, biofeedback, relajación, hipnosis y terapia cognitivo-conductual. La terapia cognitiva es usada para cambiar patrones negativos de auto derrota a actitudes que generen acciones y emociones positivas (Blackmon, 2000).

En los últimos años se le ha dado una gran importancia a los tratamientos psicológicos y al manejo de los factores psicosociales, en los dolores crónicos. El dolor crónico es un problema abrumador que puede devastar al paciente en varios niveles de su vida (familiar, social, laboral y en general en una mejor calidad de vida). El dolor crónico es más que un fenómeno físico, con repercusiones psicológicas, sociales y económicas de una enfermedad que afecta a la vida del paciente y de sus familiares.

\section{Dolor crónico y factores psicológicos}

\section{Conceptuación del dolor}

El dolor crónico es debilitante, desmoralizante y a menudo una condición abrumadora que impacta drásticamente en la vida del paciente. Algunas emociones tales como la inutilidad, miedo o ansiedad y depresión, a menudo dejan al paciente con un sentimiento de derrota. Cada año en Estados Unidos, se estima que 176,850 pacientes buscan ayuda en los centros de dolor (Market Data Enterprises: Chronic Pain Management Programs, 1995). Los pacientes referidos a clínicas contra el dolor han experimentado múltiples intervenciones muchas veces erróneas, más aún, ellos a menudo han probado todas las alternativas posibles en el último intento para encontrar ayuda profesional para curar su dolor (Barthel y Deardorff, 1998; Hubbard, Tracy y Norgan, 1996; Turk y Gatchel, 1999).

Tradicionalmente, la conceptuación del dolor se ha dado a través de un modelo biomédico: concepción lineal del fenómeno doloroso, fenómeno determinado biológicamente. Desde esta concepción lineal, el dolor ha sido conceptuado como una consecuencia o reacción de alarma ante algún daño de nuestro organismo y esta ha sido la base de los tratamientos tradicionales médicos del dolor (Díaz, Comeche y Vallejo, 2002). La falta de resultados en el tratamiento del dolor crónico, impulsa a una nueva corriente teórica biopsicosocial: el dolor es un fenómeno perceptivo cuya caracterización necesita de un enfoque multidimensional y no lineal como el enfoque biomédico. Desde esta consideración los componentes de tipo psicosocial alcanzan gran relevancia, sobre todo en los problemas de dolor crónico en donde el dolor se relaciona con el contexto social, actitudes, creencias, emociones y características específicas del dolor. La adecuada y detallada 
evaluación de dichos componentes y sus interacciones es un requisito para el diagnóstico y el tratamiento. Desde esta perspectiva, el dolor es una experiencia de tipo perceptivo que casi todo ser humano experimenta en un momento de su vida. Desde pequeños aprendemos que el dolor es una señal de alarma que nos avisa que algo anda mal en nuestro organismo. El dolor cumple una función biológica adaptativa que nos ayuda a discriminar aquellos estímulos o cosas que pueden causarnos daño o dolor. Sin embargo, el dolor no siempre cumple una función adaptativa, en muchos casos este no está relacionado con el proceso de curación de una enfermedad o de una herida. Puede no conocerse daño orgánico responsable del dolor y en estos casos se convierte en el único problema, persistiendo en el tiempo y pasando a condicionar toda la vida del paciente y de quienes se encuentran a su alrededor. En este caso se está hablando de dolor crónico, ya que a diferencia de la temporalidad característica del dolor agudo, se extiende durante largos períodos de tiempo (seis meses o más). Ó aparece o desaparece de forma recurrente sin que exista causa orgánica conocida que pueda explicarlo (Díaz, 2001). Desde el modelo psicobiológico el dolor crónico es considerado como un trastorno psicofisiológico en el que el problema se deriva de una adaptación inadecuada del sujeto a su medio (estímulos internos y externos de diferente naturaleza) y se manifiesta a través de alteraciones fisiológicas, cognitivo, conductuales y emocionales que interactúan entre ellas en diversas maneras para generar y/o perpetuar el problema (Ferrari, 2000).

\section{Características del dolor crónico de migraña}

\section{Epidemiología.}

Un estudio realizado de 1989 a 1990 en Estados Unidos, (Stephen, 2001) mostró que la prevalencia de la migraña se ha mantenido relativamente constante, aún con el crecimiento de la población. El número actual de individuos con este desorden ha aumentado de 23.6 a los 27.9 millones. Más del $18 \%$ de las mujeres estadounidenses y $6 \%$ de los hombres padecen migraña. Puede empezar en la infancia o en la adolescencia pero la prevalencia es mayor entre las edades de 25 a 55 años y declina después de éstas. Después de la pubertad, la migraña es más común en mujeres que en hombres. Esto es causado por fluctuaciones en las hormonas sexuales, comenzando con la menstruación. En México, las cifras son muy similares. Se reporta que el $12 \%$ de mujeres padece migraña y el $7.5 \%$ de los hombres (García y Nuñez, 2000).

El $48 \%$ de personas con migraña, experimentan severos dolores de cabeza por períodos de 1 a 4 días y 10\% tienen ataques por más de 20 días. Las características individuales del dolor de cabeza, pueden ser medidas en términos del dolor, síntomas asociados, limitaciones en las actividades o inhabilidad. El 53\% de los pacientes reporta el dolor acompañado con aura 
y un $91 \%$ reportaron que durante el dolor de cabeza se sentían incapaces de trabajar.

\section{Características del dolor.}

Un alto porcentaje de dolores de cabeza está comprendido dentro de dos categorías que corresponden a cefaleas de tipo tensional y cefaleas de tipo vascular, ocupando la migraña un papel sobresaliente entre las cefaleas vasculares. La migraña es un dolor crónico en donde influyen factores neurológicos, psicológicos y sociales. La migraña es definida como un recurrente dolor de cabeza con o sin aura, que dura de uno a tres días, acompañada por otros síntomas como fotofobias, náuseas, vómito y dolor de cabeza, para culminar en una siesta o sueño. Después del dolor de cabeza, puede ocurrir diarrea, fatiga y pobre concentración. Todos estos síntomas incapacitan a la persona para continuar con sus actividades cotidianas ("Headache Classification Committee of the International Headache Society", 1988). El dolor de cabeza es generalmente atribuido a muchas causas y a uno o tres mecanismos: contracción muscular, inflamación vascular o contracción o inflamación de estructuras sensitivas del dolor. La migraña es una compleja constelación de síntomas que afectan al sistema nervioso, gastrointestinal y el sistema vascular.

El dolor crónico de migraña se puede presentar con las siguientes características descritas en la Tabla 1, que plantea la "International Headache Society Migraine Classification" (1988).

Tabla 1

International Headache Society Migraine Classification

1 Migraña

1.1 Migraña sin aura

1.2 Migraña con aura

1.2.1 Migraña con aura típica

1.2.2 Migraña con aura prolongada

1.2.3 Hemiplejia típica de migraña

1.2.4 Migraña basilar

1.2.5 Migraña sin aura de dolor de cabeza

1.2.6 Migraña con principio agudo de aura

1.3 Migraña con oftalmoplejia

1.4 Migraña retinal 
1.5 Síntomas en la infancia que pueden ser precursores o asociarse con migraña.

1.5.1 Benigno paroxismal vértigo en la infancia

1.5.2 Hemiplejia en la infancia

1.6 Complicaciones de la migraña

1.6.1 Estado migrañoso

1.6.2 Infarto migrañoso

Headache Classification Committee of the International Headache Society (1988). Classification and diagnostic criteria for disorder, headache cranial neuralgias and facial pain. Cephalagia

Los ataques de migraña u otros dolores de cabeza son a menudo activados (más que causados) por uno o más factores listados en la Tabla 2 (Scharff, Turk y Marcus, 1995; Seltzer, 1982). Aunque muchos de estos activantes son comunes a la mayoría de los pacientes que experimentan migraña, cada persona parece tener un inventario único de desencadenantes que solos o juntos precipitan un ataque de migraña. La experiencia inicial universal sugiere que un primer paso en el manejo debería ser la identificación y prohibición, cuando sea relevante (Mallory, 1990). El paciente debe de contar con la siguiente información:

- Discutir el rol de los factores activantes tales como el estrés y de factores psicosociales como agravantes ocultos de la migraña. El uso de registros diarios del dolor de cabeza es útil para identificar las relaciones.

- Proveer información acerca de los factores conocidos que activan la migraña.

- Hacer una revisión de las auto observaciones del paciente y ayudarlo proporcionándole estrategias para evitar los factores activantes.

Tabla 2

Factores activantes de la migraña

Estrés emocional

Cambios en la conducta

- Pérdida de una comida: hipoglucemia

- Dormir más o menos de lo usual

- Factores ambientales

- Una luz destellante

- Mucho ruido 
- Cambios de tiempo

- Olores fuertes

- Alergenos

- Comidas y bebidas

- Chocolate

- Queso

- Carnes curadas (por ejemplo, hot dogs, tocino)

- Bebidas conteniendo cafeína

- Bebidas alcohólicas, especialmente vino tinto

- Otros factores dietéticos

- Químicos

- Asparante

- Glutanato monosódico (MSG, harina natural, proteínas, vegetales hidrolizados)

- Benceno

- Insecticidas

- Nitratos (como las conservas de carne)

- Drogas

- Atenolol

- Cafeína

- Cimetidine

- Danazol

- Diclofenaco

- H2 bloqueadores de receptores hidralazina

- Indometazin

- Nifedipine

- Nitrofurantion

- Nitroglicerina

- Anticonceptivos orales (Ethinil estradiol +)

- Reserpine

Perspectivas actuales de la migraña.

En la última década, ha habido progresos en el diagnóstico y en el manejo de la migraña, pero retos importantes se mantienen. Varias barreras pueden continuar minando los prospectos para el óptimo cuidado de los pacientes en varios círculos médicos. Por ejemplo, la migraña aún no es aceptada como un desorden médico legitimado, por que no puede ser evaluado usando pruebas objetivas, no es un tratamiento de por vida, y aunque es un dolor crónico, se asocia a eventos episódicos (Pryse-Phillips, 1998).

Por otro lado, pueden influir los prejuicios de cada paciente y la falta de credibilidad a cualquier tratamiento. Las visitas al médico son inconsistentes 
y si el médico tiene una visión muy biomédica, el paciente va a perder rápidamente la confianza al tener que estar probando uno y otro pero sin el éxito esperado (Schoenen, 1993). El reconocimiento de la inhabilidad causada por la migraña podría ayudar a legitimizar el desorden. En un reciente análisis de la Organización Mundial de la Salud sobre que tipo de trastornos inhabilita a los pacientes, se encuentra la migraña severa entre otros. Existe una mayor conciencia del impacto de la migraña en términos de reducción de estatus funcional, productividad y calidad de vida por lo que se invita a las áreas involucradas a proponer mecanismos de diagnóstico y tratamiento más efectivos (Bendtsen, 2000).

\section{Comorbilidad de la migraña.}

Existe una comorbilidad psiquiátrica y neurológica en pacientes con migraña. Esta comorbilidad, tiene implicaciones en la etiología, diagnóstico y tratamiento. En general, la asociación entre la migraña y las condiciones de comorbilidad pueden ser: 1) coincidentes, 2) causales, 3) basadas en la etiología en donde los dos desordenes están relacionados por una genética común o factores del medio; o 4) basados en la etiología donde un trastorno mental alterado o neurológico causa ambos trastornos. Ciertos trastornos psiquiátricos (depresión mayor, desorden afectivo y trastornos de ansiedad) y trastornos neurológicos (apoplejía, epilepsia y desorden del sueño) ocurren con mayor grado en pacientes con migraña (Silberstein y Lipton, 1993). Habiendo sido diagnosticada la migraña se vuelven más comunes otras condiciones asociadas con alteraciones del humor. Pacientes con depresión pueden tener alteraciones en el humor y dolor. Los clínicos necesitan considerar el problema de diagnósticos diferenciales y diagnósticos concomitantes.

En la comorbilidad de los trastornos psiquiátricos, estudios en los que correlacionan los factores psicológicos y de la personalidad con la migraña, han demostrado que las personas que padecen migraña tienen un elevado riesgo de trastornos afectivos o de ansiedad (Arena, Andrasik, y Blanchard, 1985; Holroyd, 2002, Hart, Wade y Martelli, 2003). Existe un patrón bidireccional, lo que hace pensar que la migraña no es la causa ni el resultado de la depresión. La migraña y la depresión independientemente reducen la calidad de vida relacionada con la salud (Magnusson y Becker, 2002).

La gran incidencia de migraña asociada a factores psiquiátricos y neurológicos, también se hace evidente a factores hormonales en la mujer. La migraña es hasta tres veces más común en las mujeres que en el hombre. La mayoría sufre migraña en sus años productivos de la madurez media con todo lo que implica esta etapa de vida. Se han considerado la influencia endocrinológica y de factores asociados con el ciclo reproductivo. Estrógenos y progesterona afectan la función neural de la serotonina y norepinefrina. Estos episodios casi siempre se presentan antes de la 
menstruación o tres días durante la menstruación (Kolotylo y Broomem, 2000). Por otro lado, otras líneas de investigación se han enfocado en los determinantes genéticos de la migraña y de la existencia de una hipersensibilidad para procesar información. Gerber y Schoenen (1998), reportan que existe una estructura de personalidad específica en pacientes migrañosos: son perfeccionistas, rígidos, psicológicamente inestables, ansiosos y nerviosos. En niños y niñas con migraña de 8 años de edad, los describen sus padres como más sensibles, activos, nerviosos y con pobre concentración, en comparación con sus hermanos y hermanas (Gerber, 1987). Por otro lado, se señala que existen características neuropsicológicas en los mecanismos de habituación e inhibición de respuestas o estímulos, que sugieren que induce un desgaste mayor de energía en el cerebro que provoca las migrañas. El dolor de cabeza está asociado a emociones negativas y estrés que impactan a las funciones cognitivas relacionadas con el hipotálamo y pituitaria (Hart, Wade y Martelli, 2003). Esta hipótesis de las anormalidades observadas en niños, niñas y adultos con migraña, puede ser interpretada como una posible hipersensibilidad genética a responder a los estímulos, lo cuál los hace más susceptibles al estrés. A continuación se presenta en la Figura 1, un modelo de factores que interactúan en la migraña lo cuál puede ser útil tanto para el diagnóstico como para el tratamiento (Gerber, 1998).

Figura 1. Modelo de factores de interacción en la migraña

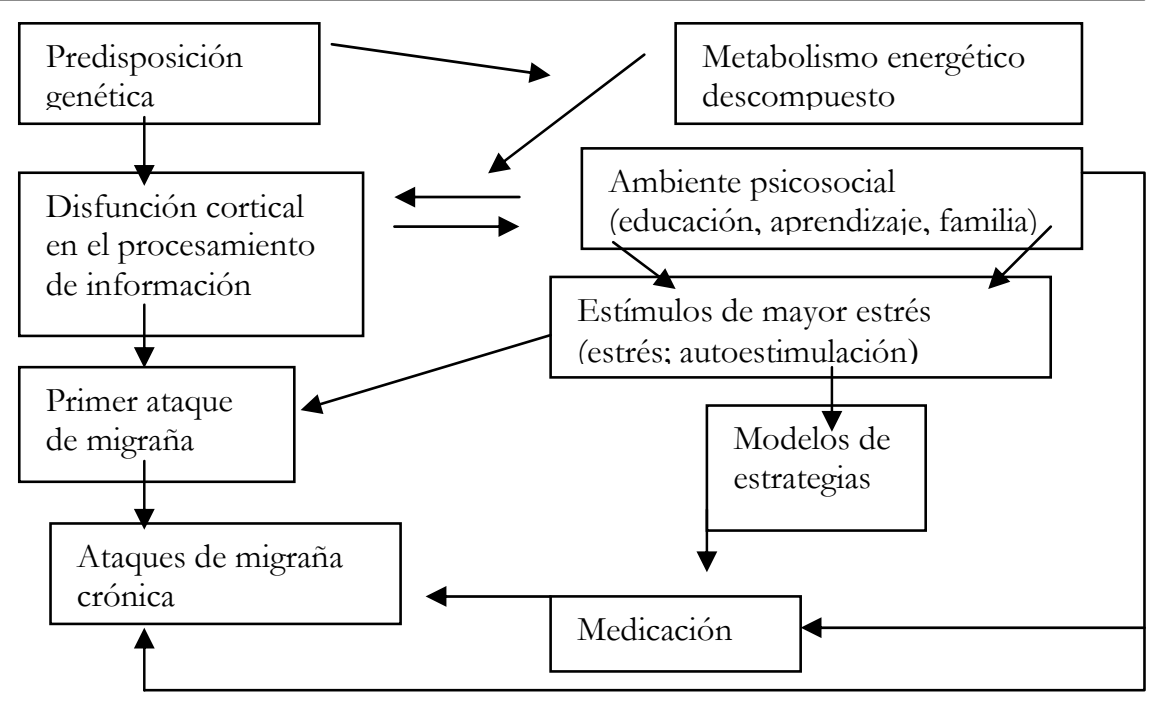




\section{Tratamiento cognitivo conductual de las migrañas}

Tradicionalmente la aproximación al estudio de la migraña fue enfocada en descripción, prevalencia, síntomas físicos, comorbilidad y la efectividad de varios tratamientos con medicamentos. Sin embargo, a pesar del largo tiempo de probar nuevos medicamentos, la migraña continúa siendo un dolor crónico que cada vez está más presente en la población. La migraña no ha sido entendida, ni evaluada ni mucho menos tratada adecuadamente de manera multicausal (Lipton y Steward 1993). A la fecha existen alrededor de 300 tipos de medicamentos y los laboratorios siguen realizando estudios sobre la eficacia de éstos, pero casi todos enfocados en el aspecto biomédico (McGrady y Andrasik, 1999).

\section{Primera aproximación.}

Antes de tomar decisiones sobre los tratamientos de la migraña, se debe tener un historial médico y un examen clínico para asegurarse que no se tiene una causa seria para el dolor de cabeza como un tumor cerebral. Posteriormente se deberá hacer una interpretación de la migraña "como un desorden psicológico, inestabilidad neuroquímica del sistema nervioso disparado por varios factores intrínsecos o extrínsecos, o ambos y el mecanismo central, vascular, periférico". La migraña es a menudo activada más que causada por uno o varios factores. Aunque muchos de los factores desencadenantes son comunes a la mayoría de las personas que experimentan migraña, cada persona parece tener un inventario que solo o juntos participan en un ataque de migraña. La experiencia clínica sugiere que un primer paso en el manejo debería ser la identificación de los activadores como estrés y factores psicosociales que agravan el dolor (Holroyd, Donnell, Stensland, Lipchik y Carlson, 2001). La identificación de los detonantes no reconocidos abre nuevas opciones de conducta para la prevención de las migrañas. Inversamente, la falta de dirección persistente de los detonantes de la migraña puede limitar la efectividad de otros tratamientos. La identificación de signos iniciales (por ejemplo, tensión muscular, claves ambientales y de estrés asociadas con el principio del dolor) pueden proveer advertencias para emplear las conductas apropiadas para el manejo de la migraña o el uso preventivo de medicamentos (Blackmon, 2000). Es importante ayudar a los pacientes a entender los dolores de cabeza y proveerles una guía clara para el uso de medicamentos. Además, los factores de conducta pueden influenciar el consumo de analgésicos. Por ejemplo, algunos pacientes toman analgésicos antes del dolor de cabeza, además ellos son capaces de predecir el inicio del dolor. Es más, a menudo por ansiedad y miedo al dolor se estimula al paciente a tomar medicinas (Lake, 2001). Para 
otros pacientes, son los efectos de los medicamentos que afectan el humor como barbitúricos o cafeína los que refuerzan el uso excesivo de los medicamentos.

\section{Métodos de observación del dolor.}

Las intervenciones conductuales pueden usarse para ayudar a los pacientes a distinguir los signos válidos de inicio del dolor de cabeza por ansiedad o miedo y pueden enseñarles técnicas alternativas para manejar la ansiedad (Holroyd, 2002). Las conductas de dolor son abiertas y así pueden observarse y registrarse. Durante los últimos quince años, la psicología cognitivo-conductual ha venido desarrollando y usando métodos de observación de las conductas del dolor como estrategias para la evaluación del paciente con dolor persistente (Keefe, 1998). El objetivo principal de la terapia cognitiva del dolor crónico de migraña, es la comprensión y modificación de las conductas del dolor. Los especialistas clínicos orientados conductualmente han desarrollado y refinado un número de estrategias para la observación de las conductas del dolor. Estas estrategias pueden ser agrupadas en dos categorías mayores: auto observación y observación directa (Keefe, 1998). En la estrategia de auto observación, se les pide a los pacientes observar y registrar sus propias conductas de dolor. Este método es práctico y no costoso para la evaluación del dolor. Un entrenamiento cuidadoso y un monitoreo continuo de los pacientes es esencial para asegurar la calidad de los datos diarios. La observación directa es a través de observadores entrenados. Se usan dos aproximaciones para la observación directa: 1) muestreo estándar de conducta y 2) observación naturalista. En el muestreo estándar de conducta se realizan registros ante varias actividades o situaciones y se correlaciona con el dolor. La observación naturalista consiste en métodos de observación estandarizados en los cuales se reproducen situaciones artificiales de la vida diaria que pueden ocasionar el dolor y se le dan estrategias conductuales para controlarlo. El uso de observaciones en el dolor crónico puede tener aplicaciones clínicas y aplicaciones en investigación. En el ámbito clínico, estos métodos podrán ofrecer datos psicológicos, sociales, físicos y del entorno del paciente para poder ofrecerle un mejor tratamiento. La evaluación detallada del dolor crónico permitirá detectar una gran cantidad de factores que pueden estar cumpliendo papeles específicos relevantes o determinantes en el dolor (Díaz, 2001). Por otro lado, en cada tipo específico de dolor crónico se deberá seleccionar el método de evaluación o de recolección de datos más apropiado (Keefe, Affeck y Lefebvre, 1997). En el ámbito de investigación, los métodos de observación pueden ser usados en estudios de investigación sobre todo en 
contextos sociales. Otra importante dirección de las investigaciones, es el análisis de la validez predictiva de las conductas observadas asociadas al dolor, así como la observación del dolor en subgrupos de pacientes con diferentes características psicológicas y sociales. Este análisis permitirá determinar qué tipo de tratamientos sería el más oportuno para cada subgrupo de pacientes.

En el dolor crónico de migraña, los métodos de observación más utilizados son: auto observación, autoinformes y cuestionarios. El que el paciente con migraña logre identificar los factores relacionados con su dolor, permitirá un mejor resultado en los tratamientos que se utilicen. Los tratamientos de conducta pueden ser eficaces sin son usados como una alternativa o junto con los medicamentos en el tratamiento de la migraña o dolor tensional tipo jaqueca. Las intervenciones más comúnmente usadas en la migraña pueden ser agrupadas en tres categorías: práctica de relajación, práctica de bioretroalimentación, y terapia cognitivo conductual (manejo de estrés). Aunque estos tratamientos intentan influenciar sobre la frecuencia y la severidad de jaquecas y migrañas, ellos hacen énfasis en la prevención de los episodios de dolor de cabeza. Los tratamientos conductuales también pueden reducir los síntomas psicológicos y de la angustia afectiva (Holroyd, 2002).

\section{Práctica de relajación.}

Las técnicas en la práctica de relajación son dirigidas para ayudar a los pacientes con migraña y jaquecas recurrentes, para ejercer control sobre el dolor relacionado con respuestas fisiológicas. La práctica de los ejercicios de relajación también asiste a los pacientes en el logro de un sentido de dominio o auto control sobre su dolor de cabeza, proporcionando una breve pausa de estrés diario y habilitando a los pacientes a manejar las situaciones estresantes diarias. Se usan tres tipos de técnicas de relajación en el tratamiento de la migraña y jaqueca: a) relajación muscular progresiva, la cuál es una relajación y tensión alternada de grupos de músculos por todo el cuerpo; b) práctica autógena, la cuál es el uso de auto afirmación de sentimientos de calidez y letargo para lograr un estado de relajación profunda; y c) ejercicios de meditación o relajación pasiva, los cuales usan palabras repetidas en silencio o sonidos para promover calma mental y relajación. Estos procedimientos de relajación a menudo son combinados en un tratamiento global. El objetivo usual de la terapia de relajación es el desarrollo a largo plazo de profilaxis, más que la reducción del dolor durante un ataque agudo. La relajación puede ser enseñada individualmente o en grupo por un médico entrenado, psicólogo u otro terapeuta. Generalmente esta técnica de relajación ha tenido mejores resultados si se combina con técnicas de biofeedback (Van-Hook, 1998; Marcus, Scharff y Turk, 1998; Arena, Hightower y Chong, 1988; Abrahamson, 1987; Fentress, Mases y Benson, 1986). 


\section{Práctica de bioretroalimentación.}

La bioretroalimentación se refiere a procedimientos que proveen información acerca de procesos fisiológicos, de manera que los pacientes puedan aprender a modificar y controlar sus respuestas introspectivas. La bioretroalimentación o "biofeedback" funciona como un espejo que le brinda al paciente la información inmediata sobre sus condiciones fisiológicas y lo capacita para aprender a modificar los comportamientos que perjudican la salud. Es un proceso de aprendizaje que requiere de un entrenamiento (usualmente a través del uso de instrumentación electrónica que monitorea las funciones vitales y las amplifica como señales acústicas o visuales, lo que permite su percepción, aprendizaje y control por parte del sujeto). Existen viarios tipos de bioretroalimentación que han sido usados sucesivamente en la profilaxis de la migraña. Una de ellas es la electromiografía (EMG) que mide la contracción y relajación muscular. Las descargas eléctricas de las neuronas motoras producen contracciones de las fibras musculares, la descarga repetida de un número significativo de neuronas motoras produce la contracción y el movimiento. El EMG, amplifica la actividad eléctrica originada en los músculos y la traduce a señales auditivas o visuales de aumento o decremento de la actividad que el paciente aprende a controlar para disminuir la tensión muscular y consecuentemente el dolor. Otra técnica es la de bioelectroencefalografía (EEG) que registra la actividad eléctrica. La descarga sincrónica de grupos de células corticales, producen ondas que son medidos por EEG, las ondas cerebrales representan potenciales oscilatorios que se categorizan en grupos básicos de frecuencia. El "biofeedback" EEG de ondas alfa sirve para ayudar a la relajación mental y desenfocar la conciencia del dolor en un procedimiento que interrumpe el ciclo. Otra técnica es la bioretroalimentación de la temperatura de la piel o de control térmico, en el cual el paciente aprende a elevar la temperatura del dedo de la mano durante las sesiones de terapia usando un dispositivo de lectura de temperatura digital. La teoría de este método es que la migraña se produce por un exceso de sangre que inunda los vasos sanguíneos de la cabeza, contraídos por un exceso de tensión nerviosa. A través de este instrumento es posible aliviar el dolor haciendo que la sangre de la cabeza circule hacia la mano, mediante un ejercicio que tiene mucho de mental: la mano se calentará, enfriándose la cabeza, bajando así la tensión sobre los vasos sanguíneos.

La bioretroalimentación es una terapia efectiva para el dolor tensional y la migraña tanto como terapia única como combinada con farmacoterapia. La combinación de retroalimentación y relajación parece ser el método más efectivo. En un estudio de 392 pacientes, se les realizó terapia de relajación, bioretroalimentación termal o las dos terapias combinadas. Los resultados indicaron que la bioretroalimentación termal fue más efectiva en disminuir la 
migraña, sin embargo, en el dolor de cabeza tipo tensional, sólo la combinación de la relajación con la retroalimentación tuvo resultados positivos (McGrady, Andrasik, Terrence, Striefel, Wickramasekera, Baskin, Penzien y Tietjen, 1999).

A pesar de que se reportan resultados positivos en el manejo de esta técnica en el dolor de cabeza, el análisis de la literatura aún no es del todo claro. Algunos estudios no encuentran diferencias con otros tratamientos, la combinación de retroalimentación con la terapia farmacológica ha sido poco estudiada. Por otro lado, la bioretroalimentación requiere un tiempo sustancial comprometido con el paciente lo cuál puede limitar su uso (Grazzi y Bussone, 1993; Arena, Bruno, Hannah y Meador, 1996; McGrady, Bush y Grubb, 1997; Arndorfer y Allen, 2001; Carpenter, 2002; Lewith, 2002). Algunas de las recomendaciones que se sugieren para una mayor efectividad del tratamiento de "biofeedback" son: determinar la disponibilidad y factibilidad de la bioretroalimentación administrada por profesionales en el cuidado de la salud entrenados/as; seleccionar pacientes para la terapia de bioretroalimentación de acuerdo a su motivación percibida y recursos financieros; considerar la bioretroalimentación como un anexo a la terapia farmacológica o como una alternativa para los pacientes intolerantes a los medicamentos y motivar la práctica y conservación de la retroalimentación en pacientes responsables.

\section{Terapia cognitiva - conductual}

La terapia cognitiva conductual para el tratamiento del dolor ha cobrado cada vez mayor relevancia en el ámbito teórico y clínico. Las intervenciones cognitivas parten de la base de que el paciente cuenta con una conceptuación inadecuada acerca de su problema de dolor, lo cual genera expectativas, creencias, actitudes, emociones y estrategias de afrontamiento desajustadas o desadaptadas que agravan el problema de dolor e impiden su recuperación. Está diseñada para ayudar a los pacientes a identificar y modificar respuestas mal adaptadas que puedan disparar o agravar la migraña. La terapia cognitiva conductual es usualmente combinada con otras terapias conductuales pero ha sido más efectiva por si sola (Holroyd, Nash y Pingel, 1991; Holroyd, 2002).

La terapia cognitiva conductual, es usada para cambiar los patrones negativos de auto derrota a otras actitudes que generen acciones y emociones positivas (Blackmon, 2002). En las intervenciones cognitivas existen técnicas de diversa naturaleza, cuya finalidad común es, frecuentemente, la de hacer referencia a procesos psíquicos no observables. Estrategias de distracción, control de la atención, imaginación, conceptuación del problema, reestructuración cognitiva de creencias y valores, entrenamiento de 
estrategias de afrontamiento, resolución de problemas, auto instrucciones, o hipnosis, son algunas de las técnicas cognitivas más usadas en el manejo del dolor crónico. El foco de intervención de estas técnicas es muy diverso, pero el objetivo de todas es tener efectos de forma indirecta sobre el dolor y sus aspectos funcionales asociados. De forma directa modifican esquemas, ideas, miedos, creencias irracionales, catastróficas, o inadecuadas, expectativas, habilidades cognitivas, percepción de auto-eficiencia, estados emocionales, sugestibilidad, o diálogo interno entre otros (Díaz, Comeche, y Vallejo, 2002).

La aproximación cognitiva-conductual para el tratamiento del dolor crónico se basa en cinco supuestos básicos: 1) las personas son procesadores activos de información en lugar que reactores pasivos a contingencias ambientales. Anticipar consecuencias se vuelve importante para guiar el comportamiento; 2) las apreciaciones, atribuciones y expectativas del individuo pueden excitar o modular factores fisiológicos que a su vez desencadenan el comportamiento y a la inversa, factores fisiológicos y psicológicos pueden influir en el pensamiento. La prioridad causal entonces, depende del punto de entrada en este ciclo de eventos interactivos; 3) el comportamiento se determina recíprocamente por el ambiente y el individuo. En un sentido muy real, la gente crea sus propios ambientes. El paciente que se vuelve consciente de un evento físico (síntoma) y decide que requiere atención médica inicia un ajuste de circunstancias diferente de aquellos de un individuo con el mismo síntoma que elige automedicarse; 4) si la gente ha aprendido formas inapropiadas de pensar, de sentir, y de responder, entonces las intervenciones exitosas para alterar el comportamiento, deben enfocarse a los pensamientos incorrectos, sentimientos, y fisiología así como los comportamientos; 5) de la misma manera en que las personas son instrumentales en el desarrollo y mantenimiento de pensamientos incorrectos, de sentimientos, de comportamientos, ellos pueden, son y deben ser considerados agentes activos en el cambio de modos incorrectos de respuesta. Pueden y deben ser participes en el aprendizaje y aplicación de modos más efectivos de respuesta al ambiente y situaciones o estados. Por lo tanto, las metas de la intervención cognitiva conductual son la conceptuación del dolor, adquisición y consolidación de habilidades, y el mantenimiento y generalización (Turk y Fernández, 1997).

La literatura que describe la estrategia cognitiva conductual para gente con dolor crónico ha crecido en la década pasada. Esta estrategia está basada en recientes desarrollos teóricos en terapias cognitivas y conductuales (Turk, Meichenbaum \& Genest, 1983). Esta enfoca ambos componentes conductuales y cognitivos del dolor crónico, enseñando a la gente con dolor crónico la relación del dolor con factores cognoscitivos, afectivos y psicológicos a fin de ayudarlos a conceptuar su propia habilidad para controlar el dolor. Las estrategias conductuales cognoscitivas enseñan 
habilidades para cambiar la forma en que la gente encara el dolor diariamente.

La estrategia es multimodal y usualmente incluye métodos de tratamiento como entrenamientos en relajación, imaginación visual, caminar, entretenimiento asertivo y fijación de objetivos, así como también práctica y consolidación de habilidades de aceptación, y otras técnicas conductuales (Kerns, Turk \& Holzman, 1983).

\section{Efectividad del tratamiento cognitivo conductual.}

Las investigaciones sobre la efectividad de la intervención cognoscitivaconductual para gente con dolor crónico se han incrementado. Asimismo, esta estrategia ha sido incorporada en la práctica de cuidados de la salud. Los artículos publicados en los primeros años de la década de los ochenta reportaron estudios de laboratorio sobre el dolor y evaluación de nuevos programas de tratamiento multidisciplinario que incluyen estrategias cognoscitivas y conductuales (Kerns et al., 1983; Turner, Clancy, McQuade \& Cardenas, 1990). En la década pasada, los resultados de los estudios han comparado la efectividad de las estrategias cognoscitivas y conductuales con otros tratamientos bien conocidos por las personas con dolor crónico (Basler, 1993; Keefe et al., 1992; Scheer, Watanabe \& Radack, 1997).

Una exploración de los avances en investigaciones y direcciones futuras en el área de las intervenciones conductuales y cognoscitivas para el dolor crónico fue conducida por Keefe et al. (1992). Ellos encontraron que los resultados de los estudios se enfocaron en la comparación de las intervenciones cognoscitivas y conductuales con condiciones de control, o prueba de la eficacia relativa de dos métodos de tratamiento, también encontraron que ninguna estrategia de tratamiento tenía una ventaja consistente sobre las otras, y sugirieron que se requieren adicionales investigaciones con poblaciones más grandes y en diferentes condiciones de dolor crónico (Keefe et al. ,1992).

Una revisión de la literatura publicada sobre la efectividad de los tratamientos cognitivos conductuales, fue realizada por Law, Stewart, Pollock, Letts, Bosch, Westmorland y Philpot, (1999). El propósito fue determinar la efectividad reportada de las intervenciones conductuales y cognoscitivas, en la mejora del desempeño ocupacional para personas con dolor crónico, mejora del desempeño de componentes ambientales y/o resultados relacionados con el dolor crónico. Una revisión crítica descriptiva incluyó todos los artículos que reportaron un estudio del efecto de las estrategias conductuales y cognoscitivas con adultos con dolor crónico. En una primera ronda, 1500 títulos con palabras clave principalmente relacionadas con las intervenciones y condiciones usadas, fueron seleccionados. Durante el proceso de revisión, muchos artículos fueron excluidos de la lista final por no cumplir con los estándares metodológicos. 
El número final de artículos aceptados por esta revisión fue de 29. De los 29 estudios incluidos en la revisión descriptiva, 13 fueron experimentales o casi experimentales en diseño. Tres de estos estudios seleccionados fueron excluidos subsecuentemente cuando ellos comparaban formas diferentes de programas de tratamiento cognoscitivo conductual. El número final de estudios seleccionados fue de 10. Los resultados de la revisión descriptiva de los 29 estudios parecen ser positivos para las estrategias cognoscitivas y conductuales en las personas con dolor crónico cuando se compararon con personas que no estaban bajo tratamiento. Sin embargo, los resultados no son positivos cuando las estrategias cognoscitivas y conductuales se comparan con métodos alternativos y otras formas de tratamiento, las estrategias del grupo de tratamiento parecen ser favorables sobre formas individuales de tratamiento. Generalmente es difícil establecer cualquier conclusión acerca de la efectividad de las estrategias cognoscitivas conductuales con la revisión descriptiva debido a que muchos estudios utilizan una metodología débil.

La revisión alternativa de 10 estudios que usaron diseños experimentales o casi experimentales encontró resultados mezclados. La mayoría de estos estudios utilizaron un diseño de control aleatorio para examinar los efectos de un programa específico de tratamiento cuando se comparó con otras formas de tratamiento (varios estudios hicieron múltiples comparaciones a la vez). Los resultados múltiples fueron evaluados, lo cual fue problemático para el análisis e interpretaciones de las revisiones sistemáticas. Sin embargo, Law, et al. (1999), encontraron en la revisión sistemática resultados favorables para las estrategas cognoscitivas y conductuales cuando se aplicaron solas y no combinadas con otros tratamientos alternativos.

En el tratamiento del dolor crónico de migraña los resultados fueron muy similares. La Agencia de Investigación para el Cuidado y Calidad de la Salud (AHCRQ) evaluó los tratamientos conductuales en los dolores crónicos de cabeza, concluyendo que éstos tratamientos han producido logros entre $40 \%$ y $50 \%$. Sin embargo, no se encontraron diferencias entre el tratamiento de bioretroalimentación y el tratamiento cognitivo conductual aplicados en forma independiente. A diferencia de otros tratamientos alternativos e inclusive de farmacología sola, estos dos tipos de tratamiento parecen ser los efectivos en el manejo del dolor crónico de migraña (Arena, et al., 1991; Llacqua, 1994; Grazzi, Amico, Leone, Moschiano y Bussone, 1998; Pryse-Phillips, 1998; Diamond, 1999; Kenneth, 2002). La práctica de la bioretroalimentación ofrece al paciente reducir el dolor de cabeza y aprender a auto controlarse y usar estrategias de relajación. Por otro lado, la terapia cognitivo conductual, enfatiza cambios en la percepción de los pacientes de que ellos pueden tomar acciones para controlar sus dolores de cabeza. Sin embargo, estos dos tratamientos tanto juntos como combinados no ofrecen diferencias significativas, tal vez por el tipo de estudios realizados, los cuales 
carecen de una metodología que nos permita asegurar la efectividad de los tratamientos. A pesar de todo la literatura es contundente en demostrar la eficacia de los tratamientos cognitivos conductuales en el manejo de dolores crónicos.

\section{Conclusiones}

La migraña exige una tremenda disminución en la calidad de vida de los individuos afectados, sus familias y la sociedad como un todo. Como un desorden crónico con manifestaciones episódicas preeminentes, la migraña es asociada con una discapacidad que socava funciones normales y los resultados en productividad son reducidos. A pesar de que se han tenido logros importantes en el manejo y control de las migrañas, aún existen severos obstáculos. Uno de ellos es que los involucrados en la salud perciben a la migraña como un "dolor no legítimo" lo que provoca que su aproximación hacia el tratamiento sea limitada.

La migraña es uno de los dolores crónicos más frecuentes de la población y esta asociado a factores neurológicos, psicológicos, de género, edad, ambientales y sociales. La migraña tiene una alta comorbilidad y su diagnóstico es esencial para determinar el manejo adecuado. Esta enfermedad es crónica como una condición a largo plazo, con intercambios entre factores sociales, biológicos y psicológicos. Con la adopción de esta perspectiva biopsicosocial se tiene un medio de evaluación, conceptuación y tratamiento del dolor crónico (Gardea y Gatchel, 2000). A pesar de que existe un gran número de medicamentos en el mercado para el tratamiento de las migrañas, el uso de la farmacología por si solo no ha tenido el impacto necesario para controlar el dolor crónico de migrañas. Desgraciadamente los clínicos de la salud que atienden este trastorno no utilizan otros métodos alternativos para el manejo del dolor crónico. Sin embargo, cada vez más se pone en evidencia el papel que juega la psicología de la salud en el diagnóstico como en el tratamiento de dolor crónico. Por un lado, la psicología cognitivo conductual ha ofrecido métodos de observación de la conducta del dolor que pueden ayudar al paciente con migraña a identificar aquellos factores relacionados o disparadores de su dolor. Por otro lado, ofrece datos de comorbilidad que se deben tomar en cuenta en el diagnóstico e intervención y por último proporciona técnicas efectivas de tratamiento en donde se toman en cuenta factores biológicos, psicológicos y sociales. En la literatura se evidencia el efecto positivo de la bioretroalimentación y de la terapia cognitivo conductual en el manejo de las migrañas. Aunque en ocasiones no contundentes, si reflejan que son tratamientos que inclusive por si solos pueden ser una buena alternativa de tratamiento. 
En futuras investigaciones es importante evaluar la efectividad de técnicas por separado, tomar en cuenta los diferentes tipos de dolores de cabeza y su comorbilidad, así como en diferentes grupos de edades (niños, adolescentes, adultos y adultos mayores) y sobre todo el uso de una metodología que permita valorar su efectividad. Por otro lado, se ha mencionado que existe un gran número de pacientes no beneficiados por las intervenciones psicológicas o tradicionales existentes y concretamente en las intervenciones cognitivo conductuales, no existe claridad sobre cuáles son los principios activos responsables del éxito de los tratamientos, tampoco sobre en qué pacientes estas intervenciones son más eficaces y en qué síndromes concretos.

Es importante trabajar en el proceso de demostrar la eficacia de estas técnicas, con el objetivo de poder competir en el sistema sanitario con los tratamientos farmacológicos. En el caso del dolor la relevancia es todavía mayor por encontrarnos ante un problema de gran incidencia y de gran impacto económico y social. La consecución de tratamientos eficaces para el control del dolor, deberá también llevar asociada una política educativa para los usuarios que los haga capaces de reclamar una intervención eficaz para su problema. Para esto se requieren programas institucionales que impliquen un cambio en la forma de conceptuar el dolor de manera biopsicosocial. El papel de psicólogos y psicólogas en el manejo de tratamientos para el dolor crónico es cada vez más crucial por lo que la creación de centros de actualización e investigación en psicología de la salud parece ser una necesidad en estos tiempos modernos.

La terapia cognitivo conductual ofrece un esperanzador futuro. El manejo interdisciplinario y un programa temprano de intervención podrían reducir una cantidad tremenda de dolor humano y sufrimiento y tal vez ahorrar billones de dólares en costo.

\section{Referencias}

Abrahamson, C.F. (1987). Response to the challenge: Effective treatment of the elderly through thermal biofeedback combined with progression relaxation. Biofeedback and Self Regulation, 12 (2), 121-125.

Arena, J., Andrasik, E. \& Blanchard, E. (1985). The role of personality in the etiology of chronic headache. Headache, 25, 296-301.

Arena, J.G., Bruno, G.M., Hannah, S.L. \& Meador K.J. (1996). A comparison of frontal electromyographic biofeedback training, trapezius electromyographic biofeedback training, and progressive muscle relaxation therapy in the treatment of tension headache. Headache, 35 (7), 411-419.

Arena, J.G., Hannah, S.L., Bruno, G.M. \& Meador, K.J. (1991). Electromyographic biofeedback training for tension headache in the elderly: A prospective study. Biofeedback and Self Regulation, 16 (4), 379-390.

Arena, J.G., Hightower, N.E. \& Chong, G.C. (1988). Relaxation therapy for tension headache in the elderly: A prospective study. Psychology and Aging, 3 (1), 96-98. 
Arndorfer, R.E. \& Allen K.D. (2001). Extending the efficacy of a thermal biofeedback treatment package to the management of tension-type headaches in children. Headache, 41 (2), 183-192.

Barthel H.R., Miller L.S. \& Deardorff W.W. (1998). Presentation and response of patient with upper extremity repetitive use syndrome to multidisciplinary rehabilitation program: Retrospective review of 24 cases. Journal Headache, 11, 191-199.

Basler, H. (1997). Incorporation of cognitive-behavioral treatment into the medical care of chronic low back patients: A controlled randomized study in german pain treatment centers. Patient Education and Counseling, 31, 113-124.

Bendtsen, L. (2000). Central sensitization in tension-type headache possible pathophysiology mechanisms. Cephalagia 20, 486-508.

Blackmon, P. (2002). Cognitive restructuring, a treatment for chronic pain. Medical Corporation Society : SPARC.

Carpenter, S. (2002). Behavioral researchers are uncovering promising new ways to treat chronic pain. Monitor on Psychology, 33 (4), 26-35.

Diamond, S. (1999). Tension-type headache. Clinical Journal Cornerstone, 1 (6), 33-44.

Díaz, M.I. (2001). Conceptualización y evaluación del dolor desde una perspectiva cognitivoconductual. Actualización en Dolor, 2 (4), 231-240.

Díaz, M.I., Comeche, M. \& Vallejo P. (2002). Tratamientos psicológicos eficaces en el dolor crónico. Psichotema, 14, 45-58.

Fentress, D.W., Masek, B.J. \& Benson, H. (1996). Biofeedback and relaxation-response training in the treatment of pediatric migraine. Developmental Medicine and Children Neurology, 28 (2), 139-146.

Ferrari, R. (2000). The biopsychosocial model -a tool for rheumatologist. Bailliéres Clinical Rheumatology, 14, 787-795.

García, P.F. \& Nuñez O.L. (2000). Epidemiología en México de la migraña. Revista Méxicana de Neurología, 37, 56-65.

Gardea, M.A. \& Gatchel, R.J. (2000). Interdisciplinary treatment of chronic pain. Current Pain and Headache Reports, 4, 18-23.

Gerber, W.D. (1987). Social and behavioral factors in children suffering from headache: The role of need for achievement and family interaction. Cephalagia 7 (7), 367-380.

Gerber, W.D. \& Schoenen J. (1998). Biobehavioral correlates in migraine: The role of hypersensitivity. Cephalalgia, 18 (21), 5-11.

Grazzi, L. \& Bussone, G. (1993). Effect of biofeedback treatment on sympathetic function in common migraine and tension-type headache. Cephalagia, 13 (3), 197-200.

Grazzi, L., Amico, D., Leone, M., Moschiano, F. \& Bussone, G. (1998). Pharmacological and behavioral treatment of pediatric migraine and tension-type headache. Italian Journal of Neurological Sciences, 19 (2), 59-64.

Hart, R.P., Wade, J.B. \& Martelli M.F. (2003). Cognitive impairment in patients with chronic pain: The significance of stress. Current Pain and Headache Reports 7, 116-126.

Holroyd, K.A. (2002). Behavioral and psychological aspects of the pathophysiology and management of tension-type headache. Current Pain and Headache Reports, 6, 401-407.

Holroyd, K.A, Nash, J.M \& Pingel, J.D. (1991). A comparison of pharmacological (amitriptyline $\mathrm{HCI}$ ) and nonpharmacologic (cognitive-behavioral) therapies for chronic tension headaches. Consulting Clinical Psychological, 59, 387-393.

Holroyd, K.A., O Donnell, D.O., Stensland, M.S., Lipchik G.L., Cardingley, M.D \& Carlson B.W. (2001). Management of chronic tension-type headache with tricyclic antidepressant medication, stress management therapy, and their combination. Journal of Consulting and Clinical Psychology, 285, 2208-2215.

Hubbard, J.E, Tracy J. \& Morgan S.F. (1996). Outcome measure of chronic pain program: A prospective statistical study. Clinical Journal Pain, 12, 330-337.

International Headache Society. (1998). Classification and diagnostic criteria for headache disorders, cranial neuralgia's and facial pain. Cephalagia, 8 (7), 13.

Jones D.R. (2000). A measure beliffattitudes about chronic non-malignant pain: A pilot study of occupational therapists. Occupational Therapy International, 7 (4), 232-245.

Kohler, T. \& Haimerl, C. (1990). Daily stress as a trigger of migraine attacks: Results of thirteen single-subject studies. Consulting Clinical Psychology, 58, 870-872. 
Keefe, F.J. (1998). Current status and future direction of pain behavior observation. Current Pain and Headache Reports, 2, 61-65.

Keefe, F.J., Dunsmore, J. \& Burnett, R. (1992). Behavioral and cognitive-behavioral approaches to chronic pain: Recent advances and future direction. Journal of Consulting and Clinical Psychology, 60, 528-536.

Kenneth, A. (2002). Behavioral and psychologic aspects of the pathophysiology and management of tension-tipe headache. Current Pain and Headache Reports, 6, 401-407.

Kerns, R.D., Turk, D.C. \& Holzman A. D. (1983). Psychological treatment for chronic pain: A selective review. Clinical Psychology Review, 3, 15-26.

Kerns, R.D., Turk, D.C \& Holzman A.D. (1986). Comparison of cognitive-behavioral and behavioral approaches to the outpatient treatment of chronic pain. The Clinical Journal of Pain, 1, 195-203.

Kleefe, F.J., Affleck, G. \& Lefebvre J.C. (1997). Coping strategies and coping efficacy in rheumatoid arthritis: A daily process analysis. Pain, 69, 43-48.

Kolotylo, C.J. \& Broome, M.E. (2000). Exploration of migraine pain, depressive sympomatology, and coping: A pilot study. Health Care for Women International, 21, 6-39.

Lake, A.E. (2001). Behavioral and nonpharmacologic treatments of headache. Journal Medical Clinics of North America, 85 (4), 1055-1075.

Law, M., Stewart, D., Pollock, N., Letts, L., Bosch, J., Westmorland, M. \& Philpot, A. (1999). The effectiveness of cognitive-behavioural interventions with people with chronic pain. A critical review of the literature by the occupational therapy evidence-based practice research group. Review Manager 3, 5-35. McMaster University, Hamilton, Ontario

Lewith, G. (2002). Treating migraine with complementary medicine. Headache, 97 (5), 273-280.

Lipton, R.B., \& Stewart W. (1993). Migraine in the United States: A view of epidemiology and health care use. Neurology, 43 (3), 6-10.

Llacqua, G.E. (1994). Migraine headaches: Coping efficacy of guided imagery training. Headache, 34 (2), 99-102.

Magnusson, J.E., \& Becker, W.J. (2002). A comparison of disability and psychological factors in migraine and transformed migraine. Cephalagia, 22, 172-178.

Mallory, D. (1990). Migraine trigger factor reduction is effective. Headache, 35 (5), 303-307.

Market Data Enterprises. (1995). Chronic pain management programs: A market analysis. New York, Valley Stream.

Marcus, D.A., Scharff, L., Mercer, S., \& Turk, D.C. (1998). Non pharmacological treatment for migraine: Incremental utility of physical therapy with relaxation and thermal biofeedback. Cephalagia, 18 (5), 266-272.

McGradt, A., Andrasik, F., \& Davies, T. (1999). Psychophysiology therapy for chronic headache in primary care. Journal of Clinical Psychiatry, 4, 96-102.

McGrady, A., Andrasik, F., Terrence, D., Striefel, S., Wickramasekera, I., Baskin, S., Penzien, B., \& Tietjen, G. (1999). Psychophysiologic therapy for chronic headache in primary care. Journal of Clinical Psychiatry, 1, 96-102.

McGrady, A.V., Bush, E.G., \& Grubb, B.P. (1997). Outcome of biofeedback-assisted relaxation for neurocardiogenic syncope and headache: A clinical replication series. Applied Psychophysiology and Biofeedback, 22 (1), 63-72.

Pryse-Phillips, D. (1998). Guidelines for the nonpharmacologic management of migraine in clinical practice. Canadian Medical Association Journal, 159, 22-39.

Scharff, L., Turk, D.C. \& Marcus D.A. (1995). Triggers of headache episodes and coping responses of headache diagnostic groups. Headache, 35, 616-626.

Scheer, Watanabe \& Radack, (1997). Prevalence of frequent headache in a population. Headache, 38, 497-506.

Schoenen, J. (1993). Exteroceptive suppression of temporaries muscle activity in patients with chronic headache and in normal volunteers: Methodology, clinical, and pathophysiologic relevance. Headache, 33, 3-17.

Schoenen, J. \& Wang, W. (1997).Tension-type headache. Headache, 177-200.

Seltzer, S. (1982). Foods, and food and drug combinations, responsible for head and neck pain. Cephalagia 3, 395-417.

Silberstein, S.D., \& Lipton, R.B. (1993). Epidemiology of migraine. Neuroepidemiology, 12, 179-194. 
Stephen, D., Silberstein, M., \& Lipton, R. (2001). National Institute of Neurological Disorders and Stroke of the National Institutes of Health. Clinical, 19 (11), 1-25.

Tellegen, A., Lykken, D., \& Bouchard, T. (1984). Personality similarity in aversive emotional states. Psychological Bulletin, 465-490.

Treed, R.D., Kenshalo, D.R., \& Gracely, R.H. (1999). The cortical representation of pain. Pain, 79, 105-111.

Turk, D., Fernández E. (1997). Cognitive-behavioral management strategies for pain and suffering. Current Pain and Headache Reports, 1, 99-106.

Turk, D., \& Gatchel, R.J. (1999). Multidisciplinary programs for rehabilitation of chronic back pain patients. Managing Low Back Pain, 2999-3011.

Turk, D., Meichenbau \& Genest, (1983). Cognitive-behavioral approach to the management of chronic pain. Currente Pain and Headache Reports, 3, 37-48.

Turner J., Clansy, S., McQuande, K. \& Cardenas, D. (1990). Effectiveness of behavioral therapy for chronic low back pain: A component analysis. Journal of Consulting and Clinical Psychology, 58 (5), 573-579.

Van-Hook, E. (1998). Non-pharmacological treatment of headaches-why?. Journal of Clinical Neuroscience, 5 (1), 43-49.

Recibido: 16 de febrero de 2004

Aceptado: 8 de noviembre de 2004 